\title{
GLIS Family Zinc Finger 1 was First Linked With Preaxial Polydactyly I in Humans by Stepwise Genetic Analysis
}

\author{
Jie-Yuan Jin ${ }^{1}$, Pan-Feng $W_{u^{2,3}}$, Fang-Mei Luo ${ }^{1}$, Bing-Bing Guo ${ }^{1}$, Lei Zeng ${ }^{2,3}$, \\ Liang-Liang Fan ${ }^{1,3,4 *}$, Ju-Yu Tang ${ }^{2,3 *}$ and Rong Xiang ${ }^{1,2,3,4 *}$
}

${ }^{1}$ School of Life Sciences, Central South University, Changsha, China, ${ }^{2}$ Department of Orthopaedics, Xiangya Hospital of Central South University, Changsha, China, ${ }^{3}$ Hunan Key Laboratory of Animal Models for Human Diseases, School of Life Sciences, Central South University, Changsha, China, ${ }^{4}$ Hunan Key Laboratory of Medical Genetics, School of Life Sciences, Central South University, Changsha, China

\section{OPEN ACCESS}

Edited by:

Andrea Del Fattore,

Bambino Gesù Children Hospital (IRCCS), Italy

Reviewed by: Jianquan Chen, Soochow University, China

Anne-Gaelle Borycki,

The University of Sheffield,

United Kingdom

${ }^{*}$ Correspondence: Liang-Liang Fan swfanliangliang@csu.edu.cn Ju-Yu Tang tangjuyu7749@163.com Rong Xiang

shirlesmile@csu.edu.cn

Specialty section: This article was submitted to Molecular and Cellular Pathology,

a section of the journal

Frontiers in Cell and Developmental

Biology

Received: 22 September 2021 Accepted: 02 December 2021

Published: 11 January 2022

Citation:

Jin J-Y, Wu P-F, Luo F-M, Guo B-B, Zeng $L$, Fan $L-L$, Tang $J-Y$ and Xiang $R$ (2022) GLIS Family Zinc Finger 1 was First Linked With Preaxial Polydactyly I in Humans by Stepwise Genetic Analysis. Front. Cell Dev. Biol. 9:781388. doi: $10.3389 /$ fcell.2021.781388
Background: Preaxial polydactyly (PPD) is one of the most common developmental malformations, with a prevalence of $0.8-1.4 \%$ in Asians. PPD is divided into four types, PPD I-IV, and PPD I is the most frequent type. Only six loci (GLI1, GL/3, STKLD1, ZRS, preZRS, and a deletion located $240 \mathrm{~kb}$ from $S H H$ ) have been identified in non-syndromic PPD cases. However, pathogenesis of most PPD patients has never been investigated. This study aimed to understand the genetic mechanisms involved in the etiology of PPD I in a family with multiple affected members.

Methods: We recruited a PPD I family (PPD001) and used stepwise genetic analysis to determine the genetic etiology. In addition, for functional validation of the identified GLIS1 variant, in vitro studies were conducted. GLIS1 variants were further screened in additional 155 PPD cases.

Results: We identified a GLIS1 variant (NM_147193: c.1061G > A, p.R354H) in the PPD001 family. In vitro studies showed that this variant decreased the nuclear translocation of GLIS1 and resulted in increased cell viability and migration. RNA sequencing revealed abnormal TBX4 and SFRP2 expression in 293T cells transfected with mutant GLIS1. Additionally, we identified a GLIS1 variant (c.664G > A, p.D222N) in another PPD case.

Conclusion: We identified two GLIS1 variants in PPD I patients and first linked GLIS1 with PPD I. Our findings contributed to future molecular and clinical diagnosis of PPD and deepened our knowledge of this disease.

Keywords: Glis1, PPD I, SFPR2, stepwise genetic analysis, nuclear localization signal, preaxial polydactyly

\section{INTRODUCTION}

Preaxial polydactyly (PPD) is one of the most common developmental malformations, occurring in 0.8-1.4\% of Asians (Evanson et al., 2016). PPD is phenotypically divided into four types: PPD I-IV, and PPD I (OMIM_174400) is the most common type in many populations (Handforth, 1950). PPD I is characterized by the duplication of one or more of the skeletal components of biphalangeal thumbs. The severity varies from a mere broadening of the distal phalanx with a slight bifurcation at 


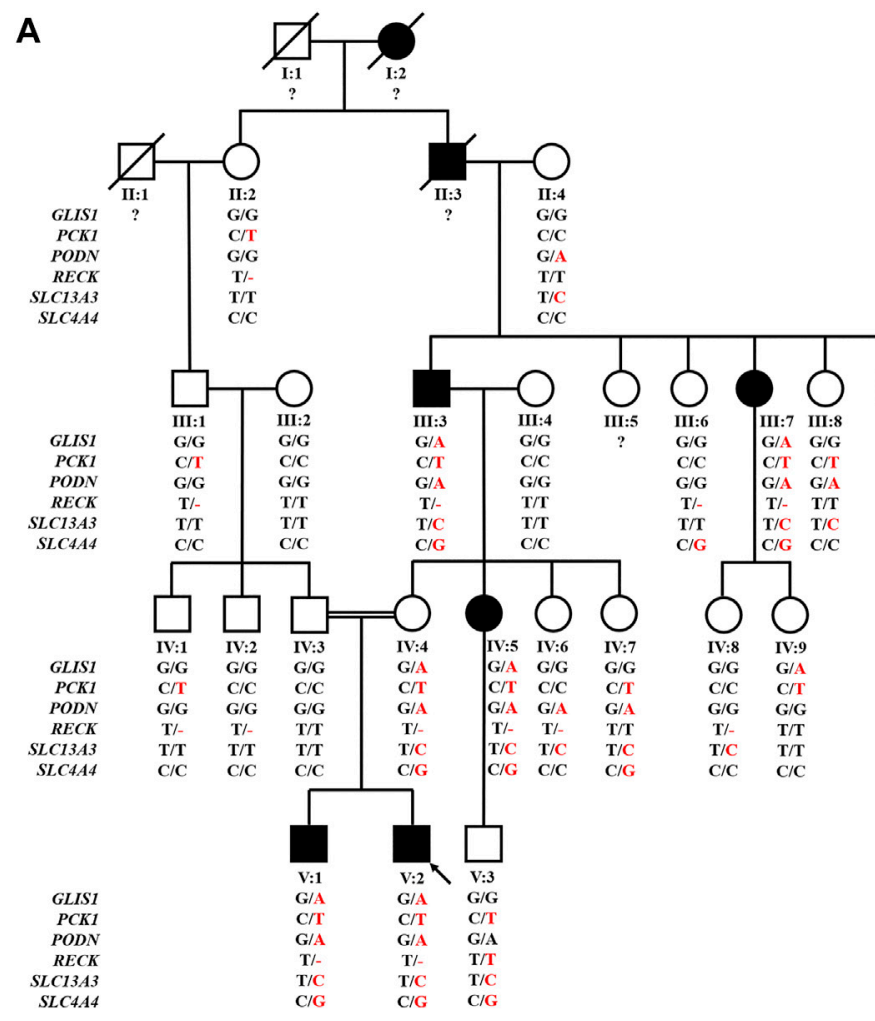

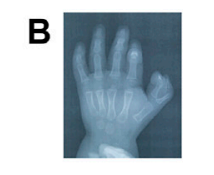

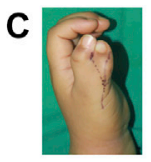

E
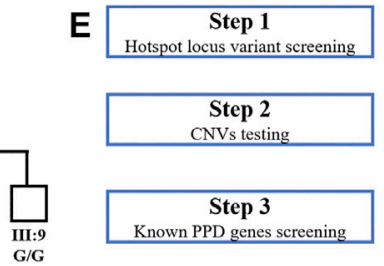

G/G

G/T

G/G
T/-
T/T

$\mathrm{T} / \mathrm{T}$
$\mathrm{C} / \mathrm{G}$
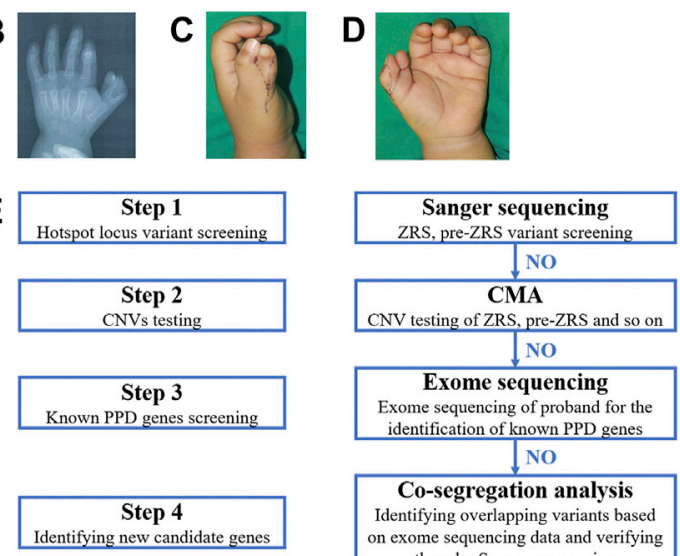
Exome sequencing of proband for the
identification of known PPD genes identification of known

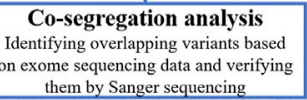

$\mathbf{F}$

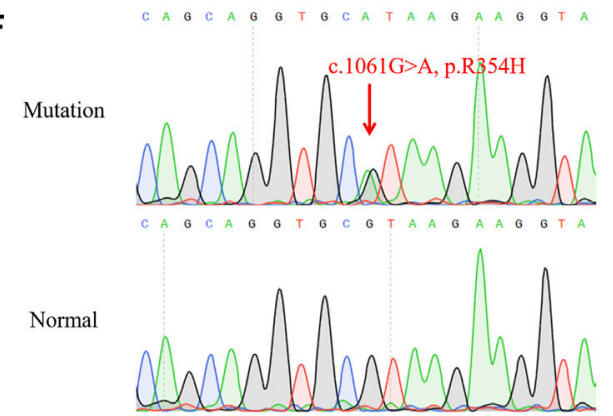

FIGURE 1 | Pedigree, symptoms, and stepwise genetic analysis of the PPD001 family. (A) Pedigree of PPD001 family with seven patients. The black symbols represent the affected members, and the arrow indicates the proband. Genotypes are identified by letters and a slash, with red letters representing variants. Question marks represent unknown data or symptoms. (B-D) The proband was affected by PPD I. (E) The stepwise genetic analysis strategy. (F) Sanger sequencing results of the GLIS1 variant (c.1061G > A, p.R354H).

the tip to a full duplication of the thumb, including the metacarpals (Lange and Muller, 2017). Currently, six loci have been identified in nonsyndromic PPD cases, including GLI1, GLI3, STKLD1, the zone of polarizing activity (ZPA) regulatory region (ZRS), which is a limb-specific enhancer of $\mathrm{SHH}$ that is located within intron five of LMBR1, the pre-ZRS region (a noncoding evolutionary conserved sequence $500 \mathrm{bp}$ upstream of the ZRS), and a deletion located $240 \mathrm{~kb}$ from the $\mathrm{SHH}$ promotor. Genetic etiologies in most PPD patients have never been evaluated (Petit et al., 2016; Potuijt et al., 2018; Ullah et al., 2019; Umair et al., 2019; Xu et al., 2020; Baas et al., 2021).

GLIS1 encodes GLIS family zinc finger protein 1, a transcriptional activator and repressor, though it primarily functions as a transcriptional activator. GLISs, together with GLIs and ZICs, constitute the Krüppel-like zinc finger family, which is characterized by classical Cys2-His2 zinc fingers (Lee et al., 2017). In situ hybridization of mouse embryos showed that Glis1 is expressed primarily in tissues with mesodermal lineages, including limb buds. Glis1 was first observed in the anteriorproximal mesenchyme during early limb bud development in mice (E9.5) and then extends along the entire apical ectodermal ridge (AER) at E11.5 (Kim et al., 2002). The expression pattern is similar to Gli3 expression, suggesting that Glis1 may play an important role in mouse limb patterning. However, Glis1 function in limb development remains ambiguous. In addition, GLIS1 is commonly used to generate human induced pluripotent stem cells (iPSCs), where GLIS1 can replace c-MYC and interact with OCT3/4, SOX2, and KLF4 to markedly enhance iPSC generation and promote multiple pro-reprogramming pathways, including NANOG, WNT, and the mesenchymal-epithelial transition (Maekawa et al., 2011). In breast cancers, GLIS1 and CUX1 cooperate to stimulate the WNT signaling pathway (Vadnais et al., 2014). GLIS1 confers susceptibility to mitral valve prolapse (MVP). The single nucleotide polymorphism (SNP), rs1879734, which is located in the first intron of GLIS1, is specifically associated with MVP (Yu et al., 2019).

Thus far, no study has reported a correlation between GLIS1 and PPD in humans. In this study, we identified a heterozygous missense variant in GLIS1 (NM_147,193: c.1061G > A, p.R354H) responsible for PPD I in a large Chinese family by stepwise genetic analysis and functional verification. This correlation was verified in other PPD cases. In summary, our study was the first to link GLIS1 variants with PPD and contributed to genetic 
screening for PPD patients, thereby deepening our knowledge of PPD.

\section{MATERIALS AND METHODS}

\section{Patients and Subjects}

This research was approved by the Review Board of Xiangya Hospital of Central South University. We recruited a fivegeneration Chinese family (PPD001) with PPD I (Figure 1A). Additional 155 PPD families or sporadic cases were recruited to screen the candidate gene. Written informed consent was obtained from the patients and their guardians. Blood was collected from the probands and blood relations. Genomic DNA (gDNA) was extracted using DNeasy Blood \& Tissue Kit (Qiagen).

\section{Polymerase Chain Reaction and Sanger Sequencing}

The sequences of ZRS regulatory region and pre-ZRS region were obtained from NCBI (https://www.ncbi.nlm.nih.gov/gene/ 105804841). Primers were designed by IDT (http://sg.idtdna. com/Primerquest/Home/Index) (Supplementary Table S1). Target sequences were amplified using polymerase chain reaction (PCR) and detected by Biosune Company Limited (Shanghai China).

\section{Chromosomal Microarray Analysis}

Chromosomal microarray analysis (CMA) was conducted in two affected individuals (V:1 and V:2 of the PPD001 family) as described by Jin et al. (2019), with slight modifications where necessary (Jin et al., 2019).

\section{Exome Sequencing and Co-Segregated Analysis}

Berry Genomics Company Limited (Chengdu, China) performed exome capture, high-throughput sequencing, and common filtering, as described previously (Jin et al., 2020). After filtering common variants (allele frequency $>0.05$ ) from the 1000G (https://www.genome.gov/27528684/1000-genomesproject/) and GnomAD (http://gnomad.broadinstitule.org), unique SNPs and insertions/deletions (indels) were identified. MutationTaster (http://www.mutationtaster.org/), PolyPhen-2 (http://genetics.bwh.harvard.edu/pph2/), and SIFT (http:// provean.jcvi.org/index.php) were used to predict variant pathogenicity. Gene function, inheritance pattern, and clinical phenotype were annotated according to OMIM (https://www. omim.org). Pathogenicity was classified according to the American College of Medical Genetics classification (Richards et al., 2015).

Eight subjects of the PPD001 family (III:3-4, III:7, IV:3-5, and $\mathrm{V}: 1-2)$ were subjected to exome sequencing. First, we screened unique SNPs that were predicted to be disease-causing. Then, we selected variants in patients (III:3, III:7, IV:5, V:1, and V:2) and IV:4, which were absent in III:4 and IV:3. We determined whether these overlapping variants were PPD causative or candidate genes (classical limb bud pattern-related genes). Finally, we selected variants that may be associated with PPD and conducted PCR and Sanger sequencing in all family members for co-segregation analysis.

\section{Mutagenesis of GLIS1-1061G > A}

pcDNA3.1-flag-GLIS1 plasmids were obtained from WZ Biosciences Inc. (Jinan, China). Mutagenesis of GLIS1-1061G $>$ A (pcDNA3.1-flag-mGLIS1) was performed using Fast Mutagenesis Kit V2 (Vazyme). GLIS1 mutant and wild-type plasmids were validated using Sanger sequencing.

\section{Cell Culture, Transfection, Transwell, and CCK8 Test}

293T cells were obtained from the National Collection of Authenticated Cell Cultures. Cells were seeded in 6-well plates $\left(5 \times 10^{5}\right.$ cells per well) and transfected with pcDNA3.1-flag-GLIS1 vectors or pcDNA3.1-flag-mGLIS1 vectors $(2 \mu \mathrm{g}$ per well) using Lipofectamine 3,000 (Invitrogen) for $24 \mathrm{~h}$, or seeded in 24 -well plates $\left(1 \times 10^{5}\right.$ cells per well) with $0.4 \mu \mathrm{g}$ vectors per well. Transwell assays using 3,000 cells were performed $24 \mathrm{~h}$ after transfection using transwell chambers (Costar). Five thousand transfected cells were seeded into a 96-well plate and cultured for $24 \mathrm{~h}$. Then, CCK8 assays were performed using CCK-8 Cell Proliferation and Cytotoxicity Assay Kit (Solarbio).

\section{Protein Extraction and Western Blot}

Nuclear and cytosolic proteins from 293T cells were extracted using Nuclear Protein Extraction Kit (Solarbio). SDS-PAGE was performed using a $12.5 \%$ PAGE Gel Rapid Preparation Kit (Yeasen). Lysates were mixed with SDS-gel sample buffer and heated at $90^{\circ} \mathrm{C}$ for $10 \mathrm{~min}$. Then, the protein samples were loaded onto the PAGE gels. After electrophoresis, the bands were electrophoretically transferred onto a nitrocellulose membrane. After blocking with $1 \%$ bovine serum albumin (BSA) in Tris-buffered saline, the membranes were incubated with anti-FLAG primary antibody (Proteintech, 20543-1-AP, 1:5,000), anti-GAPDH primary antibody (Proteintech, 104941-AP, 1:5,000), and anti-Histone-H3 polyclonal antibody (Proteintech, 17168-1-AP, 1:1,000). Chemiluminescent signals were scanned, and integrated density values were calculated with a chemiluminescent imaging system (Alpha Innotech).

\section{Immunofluorescence}

Immunofluorescence was performed on cells $48 \mathrm{~h}$ after transfection. Cells were fixed with $4 \%$ paraformaldehyde for $15 \mathrm{~min}$ and blocked in 5\% BSA in PBS for $30 \mathrm{~min}$ at room temperature. The samples were permeabilized with Triton X$100(0.15 \%)$ and incubated overnight at $4^{\circ} \mathrm{C}$ with anti-FLAG primary antibody (Proteintech, 20543-1-AP, 1:1,000) in PBS containing $5 \%$ BSA. The cells were then incubated with mouse Alexa 488-conjugated secondary antibody (Invitrogen, A-11094, 1:400) for $1 \mathrm{~h}$ at room temperature. Nuclei were stained with 
TABLE 1 | Phenotypes of parts family members in PPD001 family.

\begin{tabular}{|c|c|c|c|c|c|c|c|c|c|}
\hline $\begin{array}{l}\text { Family } \\
\text { member }\end{array}$ & I:2 & II:3 & III:3 & III:7 & IV:5 & V:1 & V:2 & IV:4 & IV:9 \\
\hline Gender & $\mathrm{F}$ & $M$ & $M$ & $\mathrm{~F}$ & $\mathrm{~F}$ & $M$ & $\mathrm{M}$ & $\mathrm{F}$ & $\mathrm{F}$ \\
\hline Age (years) & Unknown & 73 & 57 & 51 & 28 & 8 & 3 & 32 & 23 \\
\hline Preaxial polydactyly & + & Left hand & Left hand & Left hand & Left hand & Left hand & Left hand & - & - \\
\hline Triphalangeal thumb & Unknown & Unknown & - & - & - & - & - & - & - \\
\hline Other phenotypes of upper lambs & Unknown & - & - & - & - & - & - & - & - \\
\hline Lower limbs & - & - & - & - & - & - & - & - & - \\
\hline Other & Unknown & Unknown & Hypertension & Diabetes & - & - & - & - & Arrhythmia \\
\hline
\end{tabular}

F, female; M, male; +, observed phenotype; -, no symptoms.

DAPI (Solarbio), and the cells were examined using Leica SP5 confocal microscope (Leica).

\section{Total RNA Extraction, Transcriptome Sequencing, and Real-Time PCR}

Total RNA was extracted from cells $24 \mathrm{~h}$ after transfection using RNA Extraction Kit (Qiagen) and stored at $-80^{\circ} \mathrm{C}$. Transcriptome sequencing was performed by Berry Genomics Company Limited using Illumina HiSeq 4,000 sequencing platform (Illumina). Genes with $p<0.05$ and $-1<\log 2$ fold change $>1$ were considered differentially expressed genes.

Total RNA was reverse transcribed using RevertAid First Strand cDNA Synthesis Kit (Thermo) and then subjected to real-time PCR by 2xSYBR Green qPCR Mix (Thermo).

\section{Mutant Modeling}

The GLIS1 protein structure (Q8NBF1) was downloaded from SWISS-MODEL database (https://swissmodel.expasy.org/). PyMol was used to build mutant models according to the wide-type structure.

\section{Statistical Analysis}

Statistical analyses were conducted using SPSS (version 18.0; IBM, Inc.). The data were first tested for normal distribution using the Kolmogorov-Smirnov test. Differences between groups were identified using Student's $t$-test or analysis of variance (ANOVA) if the data were normally distributed, and the Mann-Whitney $U$ test or Kruskal-Wallis test was used for non-normally distributed data. Categorical data were compared using the chi-squared test.

\section{RESULTS}

\section{Clinical Features of the Five-Generation PPD I Family}

We identified a five-generation Chinese family with PPD I (PPD001, Figure 1A). The proband (V:2) was a 3-year-old boy, who was diagnosed with PPD I (Figures 1B-D). Tracing his family history, we found seven PPD I patients (including two deceased individuals) across five generations. All affected members (III:3, III:7, IV:5, V:1, and V:2) had PPD I (Table 1).
No conspicuous phenotypes were observed in the fingers or toes of other subjects.

\section{Genetic Analysis of the PPD001 Family}

Stepwise strategies were used to identify the genetic etiology of the PPD001 family (Figure 1E). We screened variants in ZRS and pre-ZRS regions by Sanger sequencing, but did not detect variants in the proband. CMA results of the proband and his brother (V:1) showed an unfavorable copy number variation (CNV; Supplementary Table S2). Then, we conducted exome sequencing to detect known PPD-related genes in the proband, but did not identify variants in these genes, indicating that there was a novel gene variant causing PPD in this family.

To further identify pathogenic variants, we selected five affected (III:3, III:7, IV:5, V:1, and V:2) and three normal family members (III:4, IV:3, and IV:4) for exome sequencing. Based on the family pedigree, we hypothesized that PPD in this family was transmitted via autosomal dominant inheritance and that IV:4 was a carrier. Thus, we screened overlapping variants in several patients and IV:4. Finally, variants in six genes were identified (Table 2). After co-segregation of all subjects, a GLIS1 variant (NM_147193: c.1061G > A, p.R354H) was retained (Figures 1A,F). Thus, we reasoned that the GLIS1 variant could be the major etiological factor in this family.

\section{Functional Analysis of the GLIS1 p.R354H Variant}

Amino acid sequence alignment analysis showed that p.R354 in GLIS1 was highly conserved throughout evolution (Figure 2A). We used UniProtKB database (https://www.uniprot.org/uniprot/ Q8NBF1) to predict that the variant p.R354H occurred in a bilateral nuclear localization signal (BNLS, Figure 2B). We hypothesized that the mutant BNLS hampered GLIS1 transportation to the nucleus and damaged GLIS1 function as a transcription factor. Wild-type GLIS1 plasmids (pcDNA-flagGLIS1) were mutated into GLIS1-1061G > A plasmids (pcDNAflag-mGLIS1, Figure 2C). Both wild-type and mutant plasmids were transfected into 293T cells. Subsequent immunofluorescence and Western blot analysis demonstrated that compared with wild-type, the mutant GLIS1 had increased cytoplasmic retention, which reduced its proportion in the 
TABLE 2 | Overlapping variants identified in PPD001 family by WES.

\begin{tabular}{|c|c|c|c|c|c|c|c|c|}
\hline Gene & Variant & $\begin{array}{l}\text { Mutation } \\
\text { taster }\end{array}$ & $\begin{array}{l}\text { PolyPhen- } \\
2\end{array}$ & SIFT & MUpro & GnomAD & $\begin{array}{c}\text { OMIM } \\
\text { clinical } \\
\text { phenotype }\end{array}$ & $\begin{array}{l}\text { American } \\
\text { College } \\
\text { of medical } \\
\text { genetics } \\
\text { classification }\end{array}$ \\
\hline GLIS1 & $\begin{array}{l}\text { NM_147193: c.1061G } \\
>\text { A, p.R354H }\end{array}$ & $D$ & $\mathrm{D} / \mathrm{B}$ & $D$ & $\begin{array}{l}\text { Decrease } \\
\text { stability }\end{array}$ & 0.00009 & - & $\begin{array}{l}\text { Likely pathogenic } \\
\text { (PS3, PP1, PP3, PP5) }\end{array}$ \\
\hline PCK1 & $\begin{array}{l}\text { NM_002591: c. } 1576 C \\
>\text { T, p.L526F }\end{array}$ & $\mathrm{D}$ & $\mathrm{D} / \mathrm{D}$ & $\mathrm{D}$ & $\begin{array}{l}\text { Decrease } \\
\text { stability }\end{array}$ & 0.00005 & $\begin{array}{l}\text { AR, Phosphoenolpyruvate } \\
\text { carboxykinase deficiency, cytosolic }\end{array}$ & $\begin{array}{l}\text { Uncertain significance } \\
\text { (PP3, PP5, BS4) }\end{array}$ \\
\hline PODN & $\begin{array}{l}\text { NM_001199080: } \\
\text { c.781G > A, p.V261M }\end{array}$ & $\mathrm{D}$ & $\mathrm{D} / \mathrm{D}$ & $\mathrm{T}$ & $\begin{array}{l}\text { Decrease } \\
\text { stability }\end{array}$ & 0.00001 & - & $\begin{array}{l}\text { Uncertain significance } \\
\text { (PP3, PP5, BS4) }\end{array}$ \\
\hline RECK & $\begin{array}{l}\text { NM_021111: } \\
\text { c.789delT, p.C263fs }\end{array}$ & $\mathrm{D}$ & $-/-$ & - & - & - & - & $\begin{array}{l}\text { Uncertain significance } \\
\text { (PM2, PP3, PP5, BS4) }\end{array}$ \\
\hline SLC13A3 & $\begin{array}{l}\text { NM_022829: c.857T > } \\
\text { C, p.M286T }\end{array}$ & $\mathrm{D}$ & $\mathrm{D} / \mathrm{D}$ & $\mathrm{D}$ & $\begin{array}{l}\text { Decrease } \\
\text { stability }\end{array}$ & 0.00011 & $\begin{array}{l}\text { AR, Leukoencephalopathy, acute } \\
\text { reversible, with increased urinary alpha- } \\
\text { ketoglutarate }\end{array}$ & $\begin{array}{l}\text { Uncertain significance } \\
\text { (PP3, PP5, BS4) }\end{array}$ \\
\hline SLC4A4 & $\begin{array}{l}\text { NM_003759: c.1435C } \\
>\text { G, p.L479V }\end{array}$ & $\mathrm{D}$ & $\mathrm{D} / \mathrm{D}$ & $D$ & $\begin{array}{l}\text { Mildly } \\
\text { decrease } \\
\text { stability }\end{array}$ & - & $\begin{array}{l}\text { AR, Renal tubular acidosis, proximal, } \\
\text { with ocular abnormalities }\end{array}$ & $\begin{array}{l}\text { Uncertain significance } \\
\text { (PM2, PP3, PP5) }\end{array}$ \\
\hline
\end{tabular}

$D$, disease causing; $T$, tolerated; $B$, benign; $A R$, autosomal recessive.

PolyPhen-2, results showed the prediction of HumDiv and HumVar in turn.

nucleus (Figures 2D,E). CCK8 and transwell assays showed that mutant GLIS1 promoted cell viability and migration (Figures 2F,G). Transcriptome sequencing data showed abnormal TBX4 and SFRP2 expression in 293T cells expressing mutant GLIS1, and these results were verified by real-time PCR (Figures 2I,J). TBX4 and SFRP2 play important roles in limb development(Ikegawa et al., 2008; Jain et al., 2018). Furthermore, p.R354H also led to alterations in the expression of other 237 genes, including BMP3, BMPER, PCDHB15, WISP1, HOXD1, and FGF3 (Figures 2I,K). Thus, we confirmed that the GLIS1 variant decreased nuclear translocation, resulting in an increase in cell viability and migration.

\section{Screening GLIS1 Variants in Sporadic or Familial Cases}

We recruited additional 155 sporadic or familial cases with PPD to further verify the correlation between GLIS1 variants and PPD. Two GLIS1 variants (c.449C > T, p.A150V and c.664G > A, p.D222N) were identified in two PPD families (PPD087 and PPD141; Figures 3A-D; Table 3). The variant (c.449C > T, p.A150V) was identified in two patients of the PPD087 family, with a minor allele frequency (MAF) of 0.00043. p.A150 was highly conserved and located in zinc finger domains (ZFDs), but bioinformatics analysis predicted that p.A150V was benign (Figures 3E,F; Table 3). Thus, it was considered a polymorphism. The variant (c.664G > A, p.D222N) arose de novo and was detected in a baby with PPD I that was conceived in vitro. The variant was predicted to be disease-causing (Table 3). p.D222 was located in ZFDs with high evolutionary conservation (Figures 3E,F). According to three-dimensional modeling of GLIS1 protein, p.D222N caused surface charge modifications that may impact the ability to bind target DNA sequences (Figure 3G). We reasoned that the GLIS1 variant (c.664G > A, p.D222N) may be the genetic etiology in the
PPD141 family, and GLIS1 may be a novel causative gene of PPD I.

\section{DISCUSSION}

PPD I is a frequent developmental defect worldwide, while disease-causing variants have only been identified in a few patients (Ullah et al., 2019; Umair et al., 2019; Xu et al., 2020). Genetic etiologies of most cases remain to be studied. In this study, we aimed to understand the genetic mechanisms involved in the etiology of PPD I in the PPD001 family through stepwise genetic analysis, a classic variant screening strategy. Six variants in six genes were detected, including GLIS1, PCK1, PODN, RECK, $S L C 13 A 3$, and SLC4A4. The PODN and SLC13A3 variants were derived from II:4. II:2 and her normal descendants (III:1, IV:1, and IV:2) harbored the PCK1 and RECK variants, but these individuals did not have limb malformations. Thus, we excluded these four candidate genes from the analysis. Moreover, five members (III:6, III:9, IV:4, IV:7, and V:3) presented with a heterozygous variant of $S L C 4 A 4$, which is responsible for isolated proximal renal tubular acidosis, an autosomal recessive disease (Igarashi et al., 1999). In contrast, only two normal members carried the GLIS1 variant (c.1061G > A, p.R354H). Given that PPD is associated with environmental effects, gender differences, and genetic factors, we speculated that the GLIS1 variant carriers without PPD (IV:4 and IV:9) may be due to individual differences, the lower possibility of PPD in females, and the variant being the main cause of PPD rather than the only cause. Hence, we determined that the GLIS1 variant was more likely to cause PPD in this family. Subsequent genetic screening in 155 cases further verified that GLIS1 was associated with PPD.

GLIS1 contains a DNA-binding domain (DBD) consisting of five Cys2-His2 ZFDs, a BNLS, and a transcription activation 


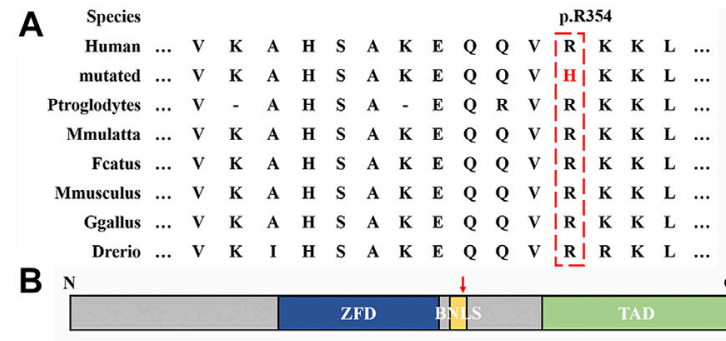

C
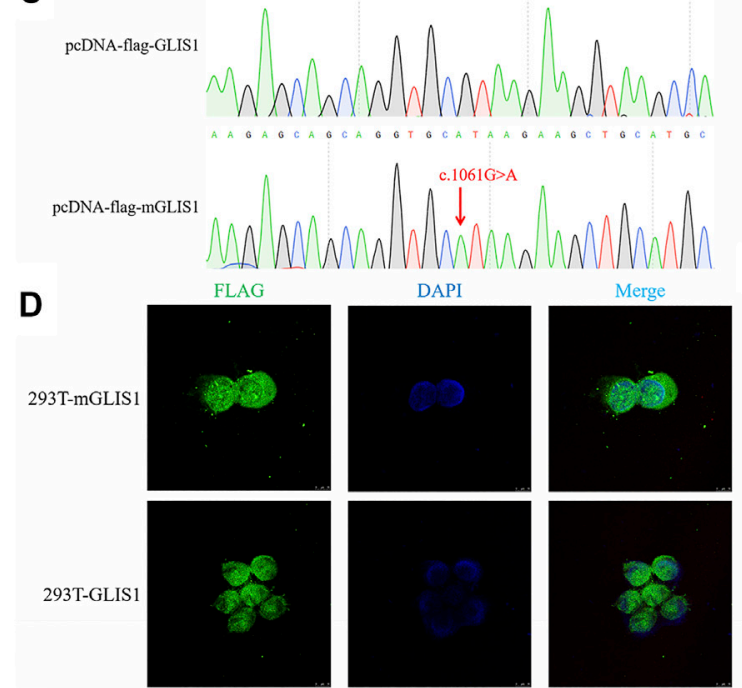

E

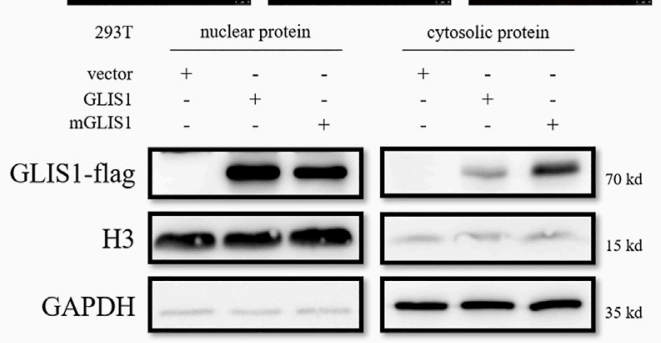

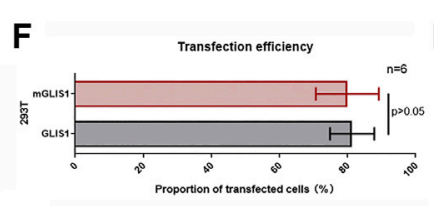

$\mathrm{H}$
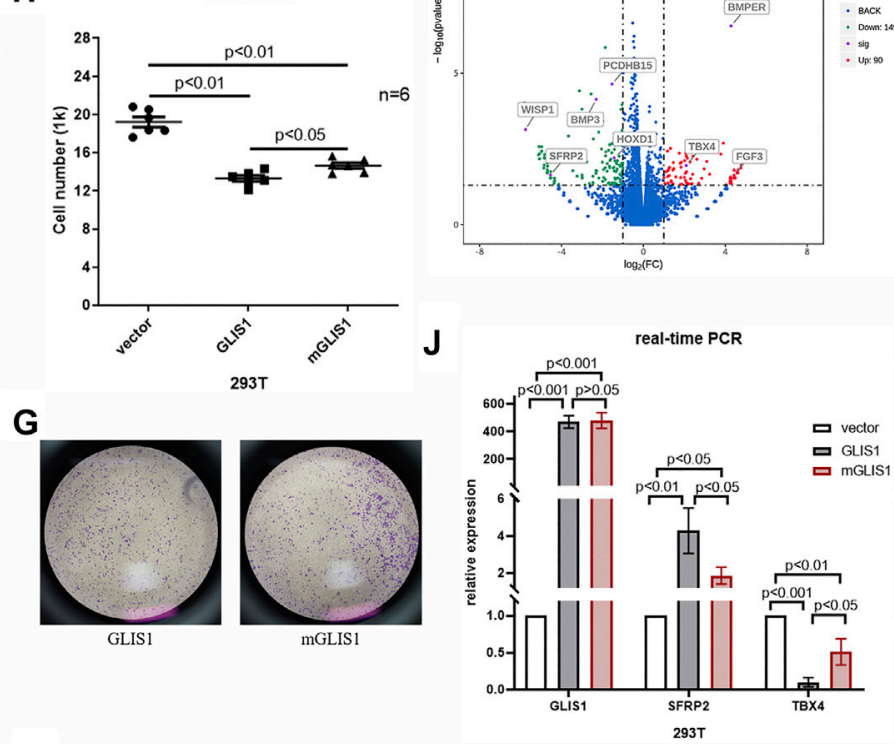

$\mathbf{K}$

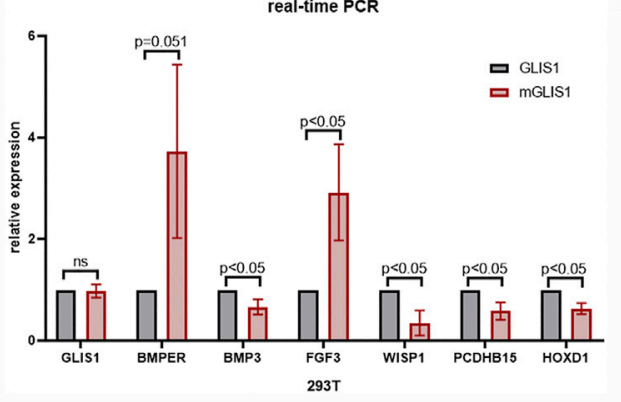

FIGURE 2 | Functional analysis of variant p.R354H in vitro. (A) Species conservation analysis of the mutant amino acid of GLIS1. (B) Schematic diagram of GLIS1 structure. Red arrow indicates variant p.R354H. (C) Sequencing results of GLIS1-1061G > A mutagenesis plasmids. (D) Immunofluorescence results demonstrating that mutant GLIS1 had increased cytoplasmic retention and reduced the proportion of nuclear protein. (E) Western blot showed that compared with wild-type, nuclear protein of the mutant GLIS1 was reduced, and cytosolic protein was increased. (F) The transfection efficiency of GLIS1/mGLIS1 plasmids in 293T cells. (G, H) CCK8 and transwell results showing that mutant GLIS1 promoted cell viability and migration. " $p<0.05$ " presents data with statistical significance, " $p>0.05$ " presents data without statistical significance, and "n" represents the number of cell samples. (I) A volcano plot based on RNA sequencing data. (J, K) Validation of RNA sequencing data by the real-time PCR. " $p<0.05$ " presents data with statistical significance, and " $p>0.05$ " presents data without statistical significance.

domain (TAD) (Jetten, 2018). Variant p.R354H was located in the BNLS and affected GLIS1 translocation to nucleus, causing cytoplasmic retention. We speculated that p.R354H may decrease GLIS1 nuclear translocation efficiency and may impede GLIS1 function as a transcription factor. Variant p.D222N occurred in ZFDs and caused the substitution of an acidic amino acid with a basic residue. Based on the threedimensional modeling results, the variant would change surface charge, which may affect the capacity of DNA binding.

GLIS1 is a regulator of mesenchymal multipotency in vitro and plays a crucial role in cell reprogramming (Scoville et al., 2017; Gerard et al., 2019). Several studies showed that GLIS1 acted on multipotent stem cells. In mouse embryos, GLIS1 is expressed primarily in the mesodermal lineages, including craniofacial regions, branchial arches, myotomes, and limb buds (Kim et al., 2002). We hypothesized that GLIS1 participated in the development of mesoblast-derived embryonic structures. PPD occurrence is accompanied by an increase in cell proliferation and migration in the anterior-proximal region of limb buds (Crick et al., 2003). Variants in GLI3 and ZRS cause PPD by inducing the anterior ectopic expression of SHH (Deng et al., 2015; Xu et al., 2020). SHH promotes cell proliferation and migration in vivo and in vitro (Hayes et al., 2001; Wang et al., 2019). We confirmed increased $293 \mathrm{~T}$ cell viability and migration after transfection with 
A

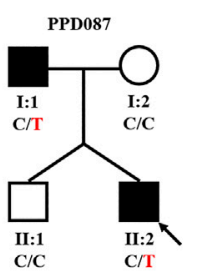

B
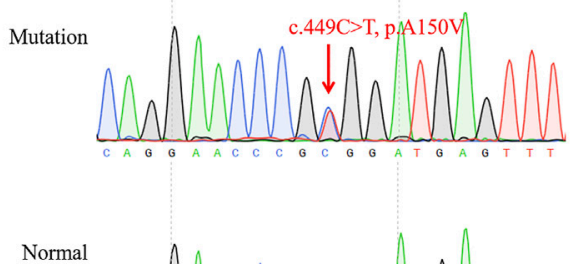

Normal

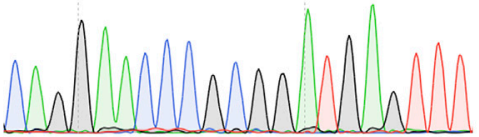

C

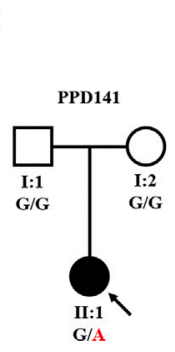

D

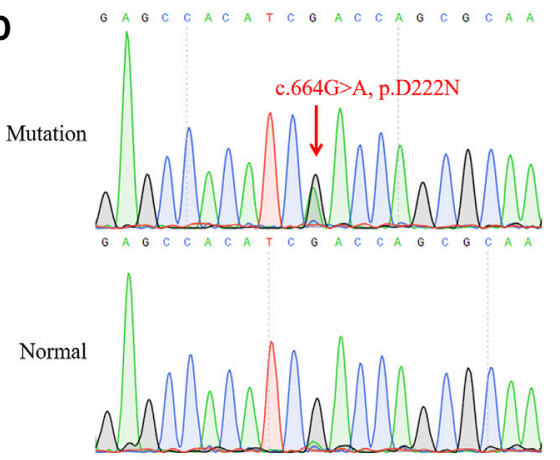

$\mathbf{F}$

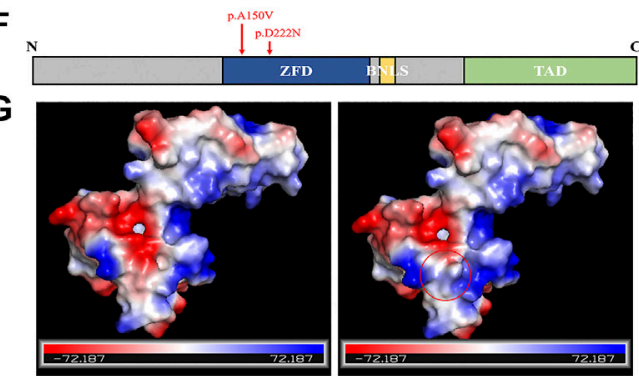

E

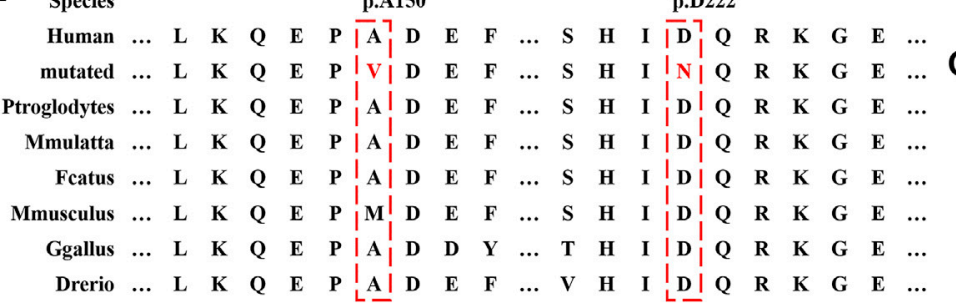

FIGURE 3 | GLIS1 variants identified in PPD I cases. (A, C) Pedigrees of PPD I families. The black symbols represent affected members, and the arrow indicates the proband. Genotypes are identified by letters and a slash, with red representing variants. (B, D) Sanger sequencing results of GLIS1 variants. (E) Species conservation analysis of mutant amino acid sites of GLIS1. (F) Schematic diagram of GLIS1 structure. The red arrows indicate variants. (G) Protein models of GLIS1 with or without the variant (p.D222N). The red circle indicates the variant site.

TABLE 3 | Phenotypes and genotypes of PPD patients with GLIS1 (NM_147193) variants.

\begin{tabular}{|c|c|c|c|c|c|c|c|c|}
\hline Patient & Phenotype & $\begin{array}{c}\text { GLIS1 } \\
\text { variant }\end{array}$ & MutationTaster & $\begin{array}{c}\text { PolyPhen- } \\
2\end{array}$ & SIFT & MUpro & GnomAD & $\begin{array}{l}\text { American } \\
\text { college } \\
\text { of medical } \\
\text { genetics } \\
\text { classification }\end{array}$ \\
\hline $\begin{array}{l}\text { PPD087-I:1 } \\
\text { PPD087- } \\
\text { II:2 }\end{array}$ & $\begin{array}{l}\text { PPD I on the right hand } \\
\text { Bilateral PPD I }\end{array}$ & $\begin{array}{l}\text { c.449C > T, } \\
\text { p.A150V }\end{array}$ & $P$ & $\mathrm{~B} / \mathrm{B}$ & $\mathrm{T}$ & $\begin{array}{l}\text { Decrease } \\
\text { stability }\end{array}$ & 0.00043 & Uncertain significance (PP1, BP4) \\
\hline $\begin{array}{l}\text { PPD141- } \\
\|: 1\end{array}$ & PPD I on the right hand & $\begin{array}{l}\text { c. } 664 G>A \\
\text { p.D222N }\end{array}$ & $\mathrm{D}$ & D/B & $\mathrm{D}$ & $\begin{array}{l}\text { Decrease } \\
\text { stability }\end{array}$ & 0.00000 & Likely pathogenic (PS2, PM2, PP3) \\
\hline
\end{tabular}

PPD I, preaxial polydactyly type I; $D$, disease causing; $T$, tolerated; $P$, polymorphism; $B$, benign.

PolyPhen-2, results showed the prediction of HumDiv and HumVar in turn.

the variant p.R354H. However, we did not observe $\mathrm{SHH}$ overexpression. Additionally, there is no report on the correlation between GLIS1 and SHH. We speculated that GLIS1 may be downstream of SHH, analogous to GLI1 and SFRP2; however, this hypothesis should be further validated (Ikegawa et al., 2008; Jeon and Seong, 2016). Sfrp2 inactivation can inhibit apoptosis in the central interdigital spaces of mice (Ikegawa et al., 2008). Decreased SFRP2 expression induced by mutant GLIS1 may better protect cells from apoptosis and may be responsible for increased cell viability in mGLIS1 293T cells.

GLIS1, GLIS2, and GLIS3 constitute a subfamily of Krüppel-like zinc finger proteins (Kang et al., 2010). As transcription factors, GLISs interact with target genes with GLIS-binding sites (GLISBS). GLISBS is a G-rich DNA sequence, (G/C)TGGGGG(A/C). The GLI and ZIC binding sites are G-rich DNA response elements similar to GLISBS (Jetten, 2018). In fact, ZFDs are highly homologous among GLISs and GLIs (Kang et al., 2010). Therefore, GLISs, GLIs, and ZICs might compete for these binding sites. For example, GLIS2 can compete with GLI1 for the same binding site as WNT4, making antagonistic action against GLI1 (Vasanth et al., 2011). Moreover, these Krüppel-like zinc finger proteins might interact and form heterodimers, such as the GLI and ZIC subfamilies (Koyabu et al., 2001). We speculated that GLIS1 can interact with GLIs or act on genes activated by GLIs during limb development. GLIs are downstream proteins of $\mathrm{SHH}$ in $\mathrm{SHH}$ signaling pathway and deeply involve in finger developments. 
SFRP2, a known SHH/GLIs target gene, was downregulated in 293T cells with mutant GLIS1 (p.R354H), hinting at the effect of GLIS1 on the SHH/GLIs signaling pathway (Milla et al., 2012). A single Sfrp2 deletion triggered syndactyly and preaxial synpolydactyly in mice, and a loss of Sfrp2 function resulted in brachy-syndactyly in mice through the Wnt signaling pathway (Ikegawa et al., 2008; Morello et al., 2008). The GLIS1 variants (c.1061G > A, p.R354H; c.664G > A, p.D222N) identified in the present study may lead to PPD by downregulating SFRP2, although there is no report about GLIS1 regulating SFPR2.

In cancers and during cellular reprogramming, GLIS1 activates the WNT signaling pathway, especially several WNTs (Maekawa et al., 2011). In this study, mutant GLIS1 upregulated BMPER, TBX4, and FGF3, while downregulating PCDHB15, BMP3, WISP1, and HOXD1. Tbx4 triggers the initiation of vertebrate limb development through activation of the Wnt/Fgf signaling cascade (Takeuchi et al., 2003). PCDHB15, WISP1, and FGF3 are involved in the WNT or FGF signaling pathways (Wong et al., 2020). BMPER regulates $\mathrm{BMP} 2 / 4$, and $\mathrm{BMP} 3$ antagonizes $\mathrm{BMP} 2$ to induce osteoprogenitor differentiation and ossification (Daluiski et al., 2001; Moser et al., 2003). BMP2 is crucial for finger outgrowth, and its variants lead to finger deformities (Dathe et al., 2009). Although WNTs did not show prominent differences in our RNA sequencing data, the expression of many WNT signaling pathwayrelated genes was altered in 293 T cells with mutant GLIS1. This may be because different cell lines have distinct expression profiles and GLIS1 target genes may be variable in different cell lines.

In this study, we detected GLIS1 variants only in two PPD I families (PPD001 family and PPD141 family). There was no evidence of a correlation between GLIS1 and other PPD types, though this conclusion required further study. The PPD001 family followed an incomplete autosomal dominant inheritance pattern, in which two GLIS1 variant carriers (IV:4 and IV:9) had no apparent limb malformations. These findings suggested that genetic factors acted in concert with environmental effects. Genome-wide association studies (GWAS) had identified GLIS1 as a susceptibility gene for MVP, but none of carriers with GLIS1 variants in this study had MVP (Yu et al., 2019).

\section{CONCLUSION}

In summary, we first associated GLIS1 with PPD I in humans by stepwise genetic analysis and examined the pathogenic potential of GLIS1 variants (c.1061G > A, p.R354H; c.664G > A, p.D222N) in vitro or by three-dimensional modeling. There are only a few known causative genes or enhancers of PPD in humans, and our research further helped us understand this disease and contributed to future molecular and clinical diagnosis of PPD. Further study should be performed to determine the potential mechanism by which GLIS1 defects cause PPD, which will be beneficial for future treatments. Given that Sfrp2 deletion is associated with limb defects in mice, and that our in vitro experiments suggested that GLIS1 can mediate SFRP2, we hypothesized that GLIS1 upregulated SFRP2 expression. Our findings hinted that GLIS1 defects may trigger reduction in SFRP2 levels to lead to PPD.

\section{DATA AVAILABILITY STATEMENT}

The original contributions presented in the study are included in the article/Supplementary Material. Further inquiries can be directed to the corresponding authors.

\section{ETHICS STATEMENT}

The studies involving human participants were reviewed and approved by the Review Board of Xiangya Hospital of the Central South University. Written informed consent to participate in this study was provided by the participants' legal guardian/next of kin.

\section{AUTHOR CONTRIBUTIONS}

JY contributed to sequencing analysis and functional verification. PW contributed to recruiting samples and collecting clinical phenotypes. LF contributed to sequencing analysis. BG contributed to variant interpretation and analysis. ZL contributed to recruiting samples and collecting clinical phenotypes. FL contributed to designing experiments and writing original draft. JT contributed to designing experiments, raising the idea of the project, and reviewing and editing the draft. RX contributed to supervision, project administration, and reviewing and editing the draft.

\section{FUNDING}

This work was supported by the National Science and Technology Major Project of the Ministry of Science and Technology of China (2017ZX10103005-006), The National Natural Science Foundation of China (81970403, 82000427, 82170598 , and 82102527), Natural Science Foundation of Hunan province (2020JJ5785), The Fundamental Research Funds for the Central Universities of Hunan Province (CX20190104), and The Emergency Project of Prevention and Control for COVID-19 of Central South University (160260003).

\section{ACKNOWLEDGMENTS}

We thank the patients and their family members for their participation in this study and all patient advisers for their assistance in clinical examination and blood specimen collection.

\section{SUPPLEMENTARY MATERIAL}

The Supplementary Material for this article can be found online at: https://www.frontiersin.org/articles/10.3389/fcell.2021.781388/ full\#supplementary-material 


\section{REFERENCES}

Baas, M., Burger, E. B., van den Ouweland, A. M., Hovius, S. E., de Klein, A., van Nieuwenhoven, C. A., et al. (2021). Variant Type and Position Predict Two Distinct Limb Phenotypes in Patients with GLI3-Mediated Polydactyly Syndromes. J. Med. Genet. 58 (6), 362-368. doi:10.1136/jmedgenet-2020106948

Crick, A. P., Babbs, C., Brown, J. M., and Morriss-Kay, G. M. (2003). Developmental Mechanisms Underlying Polydactyly in the Mouse Mutant Doublefoot. J. Anat. 202 (1), 21-26. doi:10.1046/j.14697580.2003.00132.x

Daluiski, A., Engstrand, T., Bahamonde, M. E., Gamer, L. W., Agius, E., Stevenson, S. L., et al. (2001). Bone Morphogenetic Protein-3 Is a Negative Regulator of Bone Density. Nat. Genet. 27 (1), 84-88. doi:10.1038/83810

Dathe, K., Kjaer, K. W., Brehm, A., Meinecke, P., Nürnberg, P., Neto, J. C., et al. (2009). Duplications Involving a Conserved Regulatory Element Downstream of BMP2 Are Associated with Brachydactyly Type A2. Am. J. Hum. Genet. 84 (4), 483-492. doi:10.1016/j.ajhg.2009.03.001

Deng, H., Tan, T., and Yuan, L. (2015). Advances in the Molecular Genetics of Non-syndromic Polydactyly. Expert Rev. Mol. Med. 17, e18. doi:10.1017/ erm. 2015.18

Evanson, B. J., Hosseinzadeh, P., Riley, S. A., and Burgess, R. C. (2016). Radial Polydactyly and the Incidence of Reoperation Using A New Classification System. J. Pediatr. Orthop. 36 (2), 158-160. doi:10.1097/ BPO.0000000000000395

Gérard, D., Schmidt, F., Ginolhac, A., Schmitz, M., Halder, R., Ebert, P., et al. (2019). Temporal Enhancer Profiling of Parallel Lineages Identifies AHR and GLIS1 as Regulators of Mesenchymal Multipotency. Nucleic Acids Res. 47 (3), 1141-1163. doi:10.1093/nar/gky1240

Handforth, J. R. (1950). Polydactylism of the Hand in Southern Chinese. Anat. Rec. 106 (2), 119-125. doi:10.1002/ar.1091060202

Hayes, C., Rump, A., Cadman, M. R., Harrison, M., Evans, E. P., Lyon, M. F., et al. (2001). A High-Resolution Genetic, Physical, and Comparative Gene Map of the Doublefoot (Dbf) Region of Mouse Chromosome 1 and the Region of Conserved Synteny on Human Chromosome 2q35. Genomics 78 (3), 197-205. doi:10.1006/geno.2001.6657

Igarashi, T., Inatomi, J., Sekine, T., Cha, S. H., Kanai, Y., Kunimi, M., et al. (1999). Mutations in SLC4A4 Cause Permanent Isolated Proximal Renal Tubular Acidosis with Ocular Abnormalities. Nat. Genet. 23 (3), 264-266. doi:10.1038/15440

Ikegawa, M., Han, H., Okamoto, A., Matsui, R., Tanaka, M., Omi, N., et al. (2008). Syndactyly and Preaxial Synpolydactyly in the singleSfrp2deleted Mutant Mice. Dev. Dyn. 237 (9), 2506-2517. doi:10.1002/dvdy.21655

Jain, D., Nemec, S., Luxey, M., Gauthier, Y., Bemmo, A., Balsalobre, A., et al. (2018). Regulatory Integration of Hox Factor Activity with Tbox Factors in Limb Development. Development 145 (6). doi:10.1242/dev.159830

Jeon, S., and Seong, R. H. (2016). Anteroposterior Limb Skeletal Patterning Requires the Bifunctional Action of SWI/SNF Chromatin Remodeling Complex in Hedgehog Pathway. Plos Genet. 12 (3), e1005915. doi:10.1371/ journal.pgen.1005915

Jetten, A. M. (2018). GLIS1-3 Transcription Factors: Critical Roles in the Regulation of Multiple Physiological Processes and Diseases. Cell. Mol. Life Sci. 75 (19), 3473-3494. doi:10.1007/s00018-018-2841-9

Jin, J.-Y., Wu, P.-F., He, J.-Q., Fan, L.-L., Yuan, Z.-Z., Pang, X.-Y., et al. (2020). Novel Compound Heterozygous DST Variants Causing Hereditary Sensory and Autonomic Neuropathies VI in Twins of a Chinese Family. Front. Genet. 11, 492. doi:10.3389/fgene.2020.00492

Jin, J. Y., Zeng, L., Li, K., He, J. Q., Pang, X., Huang, H., et al. (2019). A Novel Mutation (c.1010G $>$ T; p.R337L) in TP63 as a Cause of Split-hand/foot Malformation with Hypodontia. J. Gene Med. 21 (10), e3122. doi:10.1002/ jgm.3122

Kang, H. S., ZeRuth, G., Lichti-Kaiser, K., Vasanth, S., Yin, Z., Kim, Y. S., et al. (2010). Gli-similar (Glis) Krüppel-like Zinc finger Proteins: Insights into Their Physiological Functions and Critical Roles in Neonatal Diabetes and Cystic Renal Disease. Histol. Histopathol 25 (11), 1481-1496. doi:10.14670/HH25.1481
Kim, Y.-S., Lewandoski, M., Perantoni, A. O., Kurebayashi, S., Nakanishi, G., and Jetten, A. M. (2002). Identification of Glis1, a Novel Gli-Related, Krüppel-like Zinc Finger Protein Containing Transactivation and Repressor Functions. J. Biol. Chem. 277 (34), 30901-30913. doi:10.1074/jbc.M203563200

Koyabu, Y., Nakata, K., Mizugishi, K., Aruga, J., and Mikoshiba, K. (2001). Physical and Functional Interactions between Zic and Gli Proteins. J. Biol. Chem. 276 (10), 6889-6892. doi:10.1074/jbc.C000773200

Lange, A., and Müller, G. B. (2017). Polydactyly in Development, Inheritance, and Evolution. Q. Rev. Biol. 92 (1), 1-38. doi:10.1086/690841

Lee, S.-Y., Noh, H. B., Kim, H.-T., Lee, K.-I., and Hwang, D.-Y. (2017). Glis Family Proteins Are Differentially Implicated in the Cellular Reprogramming of Human Somatic Cells. Oncotarget 8 (44), 77041-77049. doi:10.18632/ oncotarget.20334

Maekawa, M., Yamaguchi, K., Nakamura, T., Shibukawa, R., Kodanaka, I., Ichisaka, T., et al. (2011). Direct Reprogramming of Somatic Cells Is Promoted by Maternal Transcription Factor Glis1. Nature 474 (7350), 225-229. doi:10.1038/ nature10106

Milla, L. A., Cortés, C. R., Hodar Q, C., Oñate, M. G., Cambiazo, V., Burgess, S. M., et al. (2012). Yeast-based Assay Identifies Novel Shh/Gli Target Genes in Vertebrate Development. BMC Genomics 13, 2. doi:10.1186/ 1471-2164-13-2

Morello, R., Bertin, T. K., Schlaubitz, S., Shaw, C. A., Kakuru, S., Munivez, E., et al. (2008). Brachy-syndactyly Caused by Loss ofSfrp2function. J. Cel. Physiol. 217 (1), 127-137. doi:10.1002/jcp.21483

Moser, M., Binder, O., Wu, Y., Aitsebaomo, J., Ren, R., Bode, C., et al. (2003). BMPER, a Novel Endothelial Cell Precursor-Derived Protein, Antagonizes Bone Morphogenetic Protein Signaling and Endothelial Cell Differentiation. Mol. Cel Biol 23 (16), 5664-5679. doi:10.1128/ MCB.23.16.5664-5679.2003

Petit, F., Jourdain, A.-S., Holder-Espinasse, M., Keren, B., Andrieux, J., Duterque-Coquillaud, M., et al. (2016). The Disruption of a Novel Limb Cis-Regulatory Element of SHH Is Associated with Autosomal Dominant Preaxial Polydactyly-Hypertrichosis. Eur. J. Hum. Genet. 24 (1), 37-43. doi:10.1038/ejhg.2015.53

Potuijt, J. W. P., Baas, M., Sukenik-Halevy, R., Douben, H., Nguyen, P., Venter, D. J., et al. (2018). A point Mutation in the Pre-ZRS Disrupts Sonic Hedgehog Expression in the Limb Bud and Results in Triphalangeal ThumbPolysyndactyly Syndrome. Genet. Med. 20 (11), 1405-1413. doi:10.1038/ gim.2018.18

Richards, S., Aziz, N., Bale, S., Bick, D., Das, S., Gastier-Foster, J., et al. (2015). Standards and Guidelines for the Interpretation of Sequence Variants: a Joint Consensus Recommendation of the American College of Medical Genetics and Genomics and the Association for Molecular Pathology. Genet. Med. 17 (5), 405-424. doi:10.1038/gim.2015.30

Scoville, D. W., Kang, H. S., and Jetten, A. M. (2017). GLIS1-3: Emerging Roles in Reprogramming, Stem and Progenitor Cell Differentiation and Maintenance. Stem Cel Investig. 4, 80. doi:10.21037/sci.2017.09.01

Takeuchi, J. K., Koshiba-Takeuchi, K., Suzuki, T., Kamimura, M., Ogura, K., and Ogura, T. (2003). Tbx5 and Tbx4 Trigger Limb Initiation through Activation of the Wnt/Fgf Signaling cascade. Development 130 (12), 2729-2739. doi:10.1242/ dev.00474

Ullah, A., Umair, M., Majeed, A. I., AbdullahJan, A., Jan, A., and Ahmad, W. (2019). A Novel Homozygous Sequence Variant in GLI1 Underlies First Case of Autosomal Recessive Pre-axial Polydactyly. Clin. Genet. 95 (4), 540-541. doi:10.1111/cge.13495

Umair, M., Bilal, M., Ali, R. H., Alhaddad, B., Ahmad, F., Abdullah, et al. (2019). Whole-exome Sequencing Revealed a Nonsense Mutation in STKLD1 Causing Non-syndromic Pre-axial Polydactyly Type A Affecting Only Upper Limb. Clin. Genet. 96 (2), 134-139. doi:10.1111/cge.13547

Vadnais, C., Shooshtarizadeh, P., Rajadurai, C. V., Lesurf, R., Hulea, L., Davoudi, S., et al. (2014). Autocrine Activation of the Wnt/ $\beta$-Catenin Pathway by CUX1 and GLIS1 in Breast Cancers. Biol. Open 3 (10), 937-946. doi:10.1242/bio.20148193

Vasanth, S., ZeRuth, G., Kang, H. S., and Jetten, A. M. (2011). Identification of Nuclear Localization, DNA Binding, and Transactivating Mechanisms of Krüppel-like Zinc Finger Protein Gli-Similar 2 (Glis2). J. Biol. Chem. 286 (6), 4749-4759. doi:10.1074/jbc.M110.165951 
Wang, X.-Z., Zhang, H.-H., Qian, Y.-L., and Tang, L.-F. (2019). Sonic Hedgehog (Shh) and CC Chemokine Ligand 2 Signaling Pathways in Asthma. J. Chin. Med. Assoc. 82 (5), 343-350. doi:10.1097/JCMA.0000000000000094

Wong, C. C., Xu, J., Bian, X., Wu, J.-L., Kang, W., Qian, Y., et al. (2020). In Colorectal Cancer Cells with Mutant KRAS, SLC25A22-Mediated Glutaminolysis Reduces DNA Demethylation to Increase WNT Signaling, Stemness, and Drug Resistance. Gastroenterology 159 (6), 2163-2180. doi:10.1053/j.gastro.2020.08.016

Xu, C., Yang, X., Zhou, H., Li, Y., Xing, C., Zhou, T., et al. (2020). A Novel ZRS Variant Causes Preaxial Polydactyly Type I by Increased Sonic Hedgehog Expression in the Developing Limb Bud. Genet. Med. 22 (1), 189-198. doi:10.1038/s41436-019-0626-7

Yu, M., Georges, A., Tucker, N. R., Kyryachenko, S., Toomer, K., Schott, J.-J., et al. (2019). Genome-Wide Association Study-Driven Gene-Set Analyses, Genetic, and Functional Follow-Up Suggest GLIS1 as a Susceptibility Gene for Mitral Valve Prolapse. Circ. Genomic Precision Med. 12 (5), e002497. doi:10.1161/ CIRCGEN.119.002497
Conflict of Interest: The authors declare that the research was conducted in the absence of any commercial or financial relationships that could be construed as a potential conflict of interest.

Publisher's Note: All claims expressed in this article are solely those of the authors and do not necessarily represent those of their affiliated organizations, or those of the publisher, the editors, and the reviewers. Any product that may be evaluated in this article, or claim that may be made by its manufacturer, is not guaranteed or endorsed by the publisher.

Copyright (c) $2022 \mathrm{Jin}, \mathrm{Wu}, \mathrm{Luo}, \mathrm{Guo}$, Zeng, Fan, Tang and Xiang. This is an openaccess article distributed under the terms of the Creative Commons Attribution License (CC BY). The use, distribution or reproduction in other forums is permitted, provided the original author(s) and the copyright owner(s) are credited and that the original publication in this journal is cited, in accordance with accepted academic practice. No use, distribution or reproduction is permitted which does not comply with these terms. 